\title{
Metabolic syndrome and risk of mortality in middle-aged versus elderly individuals: the Nord-Trøndelag Health Study (HUNT)
}

\author{
B. Hildrum • A. Mykletun • A. A. Dahl • K. Midthjell
}

Received: 7 November 2008 / Accepted: 13 January 2009/Published online: 5 February 2009

(C) Springer-Verlag 2009

\begin{abstract}
Aims/hypothesis Recent reviews indicate that the metabolic syndrome is a risk factor for cardiovascular disease and mortality, but evidence is scarce in elderly individuals. We therefore examined the relationship between the metabolic
\end{abstract}

B. Hildrum $(\bowtie)$

Department of Psychiatry, Nord-Trøndelag Hospital Trust,

Namsos Hospital,

7800 Namsos, Norway

e-mail: bjorn.hildrum@hnt.no

B. Hildrum

Department of Neuroscience, Faculty of Medicine,

Norwegian University of Science and Technology,

Trondheim, Norway

\section{A. Mykletun}

Research Centre for Health Promotion, Faculty of Psychology,

University of Bergen,

Bergen, Norway

\section{A. Mykletun}

Division of Mental Health, Norwegian Institute of Public Health, Oslo, Norway

\section{A. A. Dahl}

Department of Clinical Cancer Research,

The Norwegian Radium Hospital, Oslo University Clinic,

Oslo, Norway

\section{A. A. Dahl}

Faculty Division, The Norwegian Radium Hospital,

University of Oslo,

Oslo, Norway

\section{K. Midthjell}

HUNT Research Centre, Department of Public Health and General Practice,

Norwegian University of Science and Technology,

Verdal, Norway syndrome and mortality rates among individuals aged 40 $59,60-74$ and $75-89$ years. We also examined whether the syndrome was associated with mortality rates over and above the Framingham risk score.

Methods We studied prospectively 6,748 men and women who participated in the Nord-Trøndelag Health Study, Norway, from 1995 to 1997 (HUNT 2) and defined the metabolic syndrome by the International Diabetes Federation criteria. Results During 53,617 person-years of follow-up (mean per person, 7.9 years), 955 individuals died, of whom 585 died from cardiovascular disease. Among individuals who were 40-59 years of age at baseline, the presence of the metabolic syndrome was associated with increased relative risk of cardiovascular and total mortality (age- and sexadjusted hazard ratios 3.97 [95\% CI: 2.00-7.88] and 2.06 [1.35-3.13], respectively, equivalent to populationattributable risks of 20.7 and $14.2 \%$, respectively). The Framingham risk score accounted for less than one-third of the effect of metabolic syndrome on mortality rates. After the age of 60 years, the metabolic syndrome was not associated with increased mortality rates. We found a significant interaction between the metabolic syndrome and age on the relative risk of mortality. Results were confirmed in a subsample without cardiovascular disease at baseline. Conclusions/interpretation The metabolic syndrome is a risk factor for mortality, over and above the Framingham risk score, in middle-aged, but not in elderly individuals.

Keywords Age-specific risk - Cardiovascular disease . Epidemiology $\cdot$ Framingham risk score $\cdot$ Metabolic syndrome $\cdot$ Mortality

\section{Abbreviations}

AHA/ American Heart Association/National Heart,

NHLBI Lung and Blood Institute 


$\begin{array}{ll}\text { CVD } & \text { Cardiovascular disease } \\ \text { FRS } & \text { Framingham 10-year risk score } \\ \text { HADS } & \text { Hospital Anxiety and Depression Scale } \\ \text { HUNT } & \text { Nord-Trøndelag Health Study } \\ \text { IDF } & \text { International Diabetes Federation } \\ \text { NCEP } & \text { National Cholesterol Education Program, } \\ & \text { Adult Treatment Panel III } \\ \text { PAR } & \text { Population-attributable risk }\end{array}$

\section{Introduction}

The metabolic syndrome, defined as abdominal obesity, dyslipidaemia, hyperglycaemia and hypertension, is described as a major and increasing public health challenge worldwide [1]. Two recent reviews and one meta-analysis indicate that the syndrome is a risk factor for cardiovascular disease (CVD) incidence and mortality, as well as for total mortality [2-4]. Therefore, recently published guidelines by the International Diabetes Federation (IDF) [5] and the American Heart Association/National Heart, Lung and Blood Institute (AHA/ NHLBI) [6] recommend that all individuals found to have metabolic syndrome should receive long-term follow-up and full cardiovascular risk assessment.

Since mortality and prevalence of the metabolic syndrome are more common among elderly individuals [7], the strength of their association with increasing age is of interest. It is therefore a challenge that much of the data on the association between metabolic syndrome and mortality comes from samples consisting predominantly of younger and middle-aged individuals [2-4, 8-15], while few studies have presented age-stratified analyses. Across the studies that have addressed this association in older cohorts, increased mortality risk has not been a consistent finding [16-26]. We therefore examined the relationship between metabolic syndrome and both subsequent cardiovascular and total mortality in individuals who were stratified into three groups by age (40-59, 60-74 and 75-89 years). We also examined whether metabolic syndrome was associated with increased risk of mortality over and above a summary of conventional cardiovascular risk factors (Framingham 10 -year risk score [FRS]) across these age groups.

\section{Methods}

Participants The Nord-Trøndelag Health Study (HUNT) is a prospective, population-based study set up to cover major health issues like CVD and diabetes [27]. Briefly, all inhabitants who were 20 years or older in Nord-Trøndelag County, Norway were invited to a general health study.
Data were obtained from physical tests, blood samples and from questionnaires covering demographic characteristics, somatic illnesses, somatic and mental symptoms, medications, lifestyle and health-related behaviour. Among a total of 92,205 individuals aged 20-89 years, 65,753 (71.3\%) participated in HUNT 2 (1995-1997) [27], for 63,600 of whom data on the components of the metabolic syndrome were available. The size of the HUNT data collection made it logistically impossible to do all blood sampling after overnight fasting, but time since last meal was registered. For the purpose of this study, we included 10,206 participants $(16 \%$ of the 63,600$)$ in whom the blood samples were drawn $\geq 4 \mathrm{~h}$ after their last meal. In a prior study we had shown that this fasting sub-sample did not differ from non-fasting participants [7]. Of the included sample, another 3,458 individuals aged 20-39 years were excluded, since only 15 deaths occurred among them during follow-up, providing insufficient statistical power for analysis. This left a total study sample of 6,748 individuals aged 40-89 years at baseline. In secondary analyses, we excluded 842 individuals reporting angina pectoris (527 individuals) and/or myocardial infarction (364) and/or stroke (173) (several individuals reported more than one condition). This left 5,906 individuals in a sub-sample without self-reported CVD at baseline.

All participants in the HUNT study gave written informed consent. The Norwegian Data Inspectorate and the Regional Committee for Medical Research Ethics approved the study.

Baseline data We obtained information on use of antihypertensive and diabetes medication, known CVD and diabetes, and relevant covariates (self-reported physical activity, cigarette smoking and depression) from questionnaires. Blood pressure and waist circumference were measured at the screening site $[7,27]$. The waist circumference was measured horizontally at the height of the umbilicus with a steel band to the nearest $1.0 \mathrm{~cm}$ with the participant standing and the arms hanging relaxed. Serum analyses were performed on venous blood, using an autoanalyser (Hitachi 911; Mito, Japan). Glucose, total cholesterol and HDL-cholesterol, and triacylglycerol were measured by an enzymatic hexokinase method, an enzymatic colorimetric cholesterolesterase method and an enzymatic colorimetric method respectively. Diabetes was defined as self-reported, known diabetes, i.e. self-reported in answer to the question 'Do you or have you had diabetes?' Those confirming a diagnosis of diabetes were re-invited to provide another blood sample in the fasting state with collection of glucose, C-peptide and antibodies to glutamic acid decarboxylase. Based on this and on information on when insulin treatment was initiated, type 1 and type 2 diabetes were diagnosed [7, 27]. 
We defined metabolic syndrome according to the IDF criteria [5], which require central obesity (defined as waist circumference $\geq 94 \mathrm{~cm}$ for men and $\geq 80 \mathrm{~cm}$ for women in Europids) plus at least two of four components: serum triacylglycerol $>1.7 \mathrm{mmol} / 1$ or specific treatment for this lipid abnormality; HDL-cholesterol $<1.03 \mathrm{mmol} / 1$ in men and $<1.29 \mathrm{mmol} / 1$ in women or specific treatment for this lipid abnormality; blood pressure $\geq 130 / \geq 85 \mathrm{mmHg}$ or treatment for previously diagnosed hypertension; and fasting plasma glucose $\geq 5.6 \mathrm{mmol} / 1$ or previously diagnosed type 2 diabetes. We had no data on specific treatment for lipid abnormalities, but such treatment was uncommon in Norway at that time. Before further analyses, we adjusted triacylglycerol and glucose levels for participants who had fasted for 4, 5, 6, 7 or $8 \mathrm{~h}$ when sampled, with those reporting $\geq 9 \mathrm{~h}$ fasting as reference group [7].

Four potentially confounding factors were included in the multivariate analyses. Low physical activity was defined as less than $1 \mathrm{~h}$ leisure time physical activity per week and smoking as current cigarette smoking [28]. Total cholesterol was included as a continuous variable. As depression is known to increase CVD and all-cause mortality rates, the Hospital Anxiety and Depression Scale (HADS) ratings were included with the conventional caselevel for depression ( $\geq 8$ out of 21 points on the depression subscale) [29].

Follow-up and outcome measures Baseline examinations were from August 1995 to June 1997 and follow-up was concluded on 31 December 2004. We used the Norwegian National Death Registry and defined the outcomes as death from CVD (codes I00-I99 in the International Classification of Diseases, tenth revision, as the underlying or a contributing cause of death) and death from any cause. In a supplementary analysis, we narrowed the definition of death from CVD to include CVD only as the underlying diagnosis.

Statistical methods Descriptive statistics of included variables were examined for the three age groups. For each age group, we used Cox proportional hazard models adjusted for age (continuous) and sex to examine the association of metabolic syndrome and its components with the relative risk of CVD and all-cause mortality. The curves representing the cumulative survival rates were estimated using the Kaplan-Meier method. We also examined the association between metabolic syndrome and mortality rates after further adjustments for other risk factors (physical activity, smoking, total cholesterol and depression).

Second, we examined the association between metabolic syndrome and mortality rates in the sub-sample without self-reported CVD at baseline. An FRS was calculated for each person, using the sex-specific prediction formulas based on conventional cardiovascular risk factors (age, HDL-cholesterol, total cholesterol, blood pressure and smoking status), as modified by the AHA/NHLBI [30]. In separate analyses we examined the additional risk of metabolic syndrome over and above these factors by adjusting for the FRS.

Third, in addition to estimates of relative risk (HR) from Cox models, we calculated the proportion of mortality which theoretically would be prevented if the metabolic syndrome could be eliminated, assuming a causal relationship. This was done by including estimates of population-attributable risks (PARs), which were calculated from the formula $\mathrm{P}(\mathrm{E}) \times$ $(\mathrm{HR}-1) / \mathrm{HR}$, where $\mathrm{P}(\mathrm{E})$ indicates prevalence of exposure (metabolic syndrome).

We tested for effect modification by age (the three age groups) by adding the interaction term age by metabolic syndrome to a Cox model comprising metabolic syndrome, age and sex to predict CVD and all-cause mortality rates. Similarly, we tested for interaction between age and each syndrome component, and then for interaction between metabolic syndrome and age in the sub-sample without CVD. Significance for interaction was reported as change in step $\chi^{2}$ test value. Corresponding analyses of interactions for sex were performed. As we found no metabolic syndrome by sex interaction either in the three age groups or in the entire sample or in the sub-sample without selfreported CVD (all $p>0.33$ ), all results are reported for men and women together.

In supplementary analyses, we evaluated whether the metabolic syndrome was associated with increased relative risk of non-CVD mortality (all other causes of deaths). Finally, we repeated the multivariate analyses using the revised (2005) National Cholesterol Education Program, Adult Treatment Panel III (NCEP) criteria for metabolic syndrome [6], which define higher cut-offs for waist circumference ( $>102 \mathrm{~cm}$ in men, $>88 \mathrm{~cm}$ in women) than the IDF criteria. Again, we also tested for effect modification by age.

A two-sided $p$ value of $<0.05$ was considered statistically significant. We used SPSS software (version 14.0; SPSS, Chicago, IL, USA) for all analyses.

\section{Results}

Baseline characteristics of the participants are shown in Table 1. Prevalence of the metabolic syndrome increased with age, as did that for its components. During 53,617 person-years (mean follow-up, 7.9 years, maximum 9.5 years), 955 individuals died, of whom 585 died from CVD. In the sub-sample without self-reported CVD at baseline, 650 died, 352 from CVD (Table 2). 
Table 1 Baseline characteristics of the study participants

\begin{tabular}{|c|c|c|c|}
\hline \multirow[t]{2}{*}{ Characteristics } & \multicolumn{3}{|l|}{ Age groups (years) } \\
\hline & $40-59(n=3,789)$ & $60-74(n=1,973)$ & $75-89(n=986)$ \\
\hline Male sex $(\%)$ & 50 & 50 & 44 \\
\hline Metabolic syndrome (\%) & 28 & 47 & 57 \\
\hline \multicolumn{4}{|l|}{ Components of metabolic syndrome } \\
\hline Waist circumference, men $(\mathrm{cm})$ & $93(9)$ & $95(10)$ & $96(10)$ \\
\hline Waist circumference, women (cm) & $82(12)$ & $87(12)$ & $88(11)$ \\
\hline Systolic blood pressure (mmHg) & $136(18)$ & $154(23)$ & $162(25)$ \\
\hline Diastolic blood pressure $(\mathrm{mmHg})$ & $84(11)$ & $87(13)$ & $87(14)$ \\
\hline Triacylglycerol (mmol/l) & $1.50(1.18)$ & $1.68(1.03)$ & $1.71(1.02)$ \\
\hline HDL-cholesterol, men (mmol/1) & $1.26(0.34)$ & $1.30(0.38)$ & $1.24(0.40)$ \\
\hline HDL-cholesterol, women (mmol/l) & $1.55(0.40)$ & $1.52(0.43)$ & $1.44(0.41)$ \\
\hline Plasma glucose (mmol/l) & $5.44(1.01)$ & $5.79(1.30)$ & $6.12(1.75)$ \\
\hline Type 2 diabetes $(\%)$ & 1.1 & 3.6 & 6.5 \\
\hline Antihypertensive medication (\%) & 8.3 & 27.2 & 31.9 \\
\hline \multicolumn{4}{|l|}{ Other risk factors } \\
\hline Low physical activity ${ }^{\mathrm{a}}(\%)$ & 30 & 41 & 64 \\
\hline Current smoking ${ }^{\mathrm{a}}(\%)$ & 37 & 25 & 12 \\
\hline Total cholesterol (mmol/l) & $6.13(1.14)$ & $6.66(1.26)$ & $6.52(1.31)$ \\
\hline Depression $^{\mathrm{a}}(\%)$ & 11 & 13 & 16 \\
\hline $\mathrm{CVD}^{\mathrm{b}}(\%)$ & 4 & 19 & 31 \\
\hline Framingham risk score $^{\mathrm{c}}$ & $12.1(4.5)$ & $16.6(3.0)$ & $19.2(3.4)$ \\
\hline
\end{tabular}

Data are mean $(\mathrm{SD})$ or percentage

${ }^{a}$ Self-reported. Low physical activity was defined as less than $1 \mathrm{~h}$ leisure time activity per week. Depression was assessed by the HADS and defined as a score of $\geq 8$ out of 21 on the depression subscale. Data on depression were missing in $8.8 \%$ of the participants

${ }^{\mathrm{b}}$ Self-reported angina pectoris, myocardial infarction and/or stroke

${ }^{c}$ FRS using sex-specific prediction formulas based on age, total cholesterol and HDL-cholesterol, blood pressure and smoking [30] in the subsample without self-reported CVD at baseline $(n=5,906)$

Metabolic syndrome and mortality in the total sample In models adjusted for age (continuous) and sex, the metabolic syndrome was associated with a fourfold increased relative risk of CVD mortality and a twofold increased relative risk of total mortality in participants aged 40-59 years at baseline (Table 2). After the age of 60 years, the metabolic syndrome was not significantly associated with higher relative risk of mortality (all HRs for CVD and all-cause mortality were within range 1.05-1.12). We found a significant interaction between metabolic syndrome and age on the relative risk of CVD and all-cause mortality ( $p \leq$ 0.006 for interactions). The findings are illustrated in the Kaplan-Meier estimates of the rates of CVD and all-cause mortality according to metabolic syndrome (Fig. 1). In the youngest age group, the estimated PAR for CVD mortality associated with the metabolic syndrome was $20.7 \%$, compared with much lower proportions in the older age groups (3.1 and 4.6\%, respectively). However, the PARs in the older age groups were all derived from non-significant HRs.

Abdominal obesity was the only component of the metabolic syndrome that was significantly associated with increased relative risk of mortality in participants aged 40
59 years at baseline, with HR 3.18 (95\% CI: 1.49-6.81) for CVD mortality rates and HR $1.71(1.11-2.63)$ for total mortality rates. After the age of 60 years the corresponding HRs were not significant. All other components were associated with increased relative risk of mortality in the expected directions, but findings were not statistically significant. We also found that relative risk of mortality tended to decline with increasing age for all components; the age by component interaction was significant for effect of abdominal obesity on relative risk of CVD mortality $(p=0.027)$.

Adjustment for potentially confounding factors in the multivariate analyses did not substantially change the effect of metabolic syndrome on the risk of mortality (Table 2). The syndrome remained an independent risk factor for CVD and all-cause mortality in participants aged 40 to 59 years at baseline (both $p<0.001$ ), but not in participants aged 60 years and above (all $p>0.22$ ).

Metabolic syndrome and mortality in the sub-sample without self-reported CVD The declining relative risks with increasing age were confirmed when we restricted the analyses to the sub-sample without self-reported CVD at 
Table $2 \mathrm{CVD}$ and all-cause mortality according to presence or absence of the metabolic syndrome in the total sample and in the sub-sample without self-reported CVD at baseline

\begin{tabular}{|c|c|c|c|c|c|c|}
\hline \multirow[b]{2}{*}{ Variable } & \multicolumn{3}{|l|}{ CVD mortality } & \multicolumn{3}{|l|}{ All-cause mortality } \\
\hline & $40-59$ years & 60-74 years & $75-89$ years & $40-59$ years & $60-74$ years & $75-89$ years \\
\hline \multicolumn{7}{|l|}{$\begin{array}{l}\text { Total sample } \\
(n=6,748)\end{array}$} \\
\hline $\begin{array}{l}\text { Deaths during follow-up, } n \\
\text { Metabolic syndrome }\end{array}$ & 35 & 219 & 331 & 88 & 364 & 503 \\
\hline Age $^{\mathrm{a}}$ - and sex-adjusted HR & $3.97(2.00-7.88)$ & $1.07(0.82-1.39)$ & $1.12(0.90-1.40)$ & $2.06(1.35-3.13)$ & $1.10(0.90-1.36)$ & $1.05(0.88-1.25)$ \\
\hline $\operatorname{PAR}(\%)^{\mathrm{b}}$ & 20.7 & 3.1 & 4.6 & 14.2 & 4.3 & 2.1 \\
\hline Multivariable $\mathrm{HR}^{\mathrm{c}}$ & $3.95(1.96-7.97)$ & $1.09(0.83-1.43)$ & $1.11(0.89-1.39)$ & $2.14(1.40-3.29)$ & $1.14(0.92-1.41)$ & $1.05(0.87-1.26)$ \\
\hline \multicolumn{7}{|l|}{$\begin{array}{l}\text { No self-reported CVD } \\
(n=5,906)\end{array}$} \\
\hline $\begin{array}{l}\text { Deaths during follow-up, } n \\
\text { Metabolic syndrome }\end{array}$ & 21 & 142 & 189 & 72 & 264 & 314 \\
\hline Age $^{\mathrm{a}}$ - and sex-adjusted HR & $6.35(2.46-16.4)$ & $1.15(0.82-1.61)$ & $0.98(0.74-1.31)$ & $2.03(1.28-3.23)$ & $1.15(0.90-1.47)$ & $0.95(0.76-1.19)$ \\
\hline $\operatorname{PAR}(\%)^{\mathrm{b}}$ & 22.6 & 6.0 & $<0$ & 13.6 & 6.0 & $<0$ \\
\hline Framingham adjusted $\mathrm{HR}^{\mathrm{d}}$ & $4.61(1.74-12.2)$ & $1.07(0.75-1.51)$ & $1.02(0.75-1.37)$ & $1.63(1.01-2.63)$ & $1.09(0.84-1.41)$ & $1.03(0.82-1.30)$ \\
\hline $\operatorname{PAR}(\%)^{\mathrm{d}}$ & 21.0 & 3.0 & 1.1 & 10.3 & 3.8 & 1.6 \\
\hline
\end{tabular}

Unless otherwise stated, values are HR $(95 \% \mathrm{CI})$

${ }^{a}$ Continuous within each age group

${ }^{\mathrm{b}}$ Adjusted for age (continuous within each age group) and sex

${ }^{\mathrm{c}}$ Adjusted for age (continuous within each age group), sex, low physical activity, current smoking, total cholesterol and depression

${ }^{\mathrm{d}} \mathrm{HR}$ and PAR (\%) adjusted for FRS

baseline (Table 2). Again, metabolic syndrome was a risk factor for mortality only in the youngest age group, with HR 6.35 (95\% CI: 2.46-16.4) for CVD mortality and HR $2.03(1.28-3.23)$ for all-cause mortality. We found a significant interaction between metabolic syndrome and age on the relative risk of CVD $(p<0.001)$ and of all-cause
( $p=0.013$ ) mortality. After adjusting for FRS, the relative mortality risks associated with the metabolic syndrome remained elevated in the youngest age group (Table 2).

Supplementary analyses First, we found no significant effect of metabolic syndrome on the relative risk of non-
Fig. 1 Kaplan-Meier estimates of the rates of CVD $(\mathbf{a}-\mathbf{c})$ and all-cause (d-f) mortality by age groups 40-59 (a, d), 60-74 (b, e) and 75-89 (c, f) years according to the metabolic syndrome. $p$ values are for presence (continuous lines) vs absence (dotted lines) of the metabolic syndrome, estimated with Cox models adjusted for age (continuous within each group) and sex. $p<0.001$ (a), $p=0.64$ (b), $p=0.31$ (c), $p=0.001$ (d), $p=0.36$ (e), $p=0.61$ (f) Note: panels have different scales for cumulative survival due to differences in mortality rates a

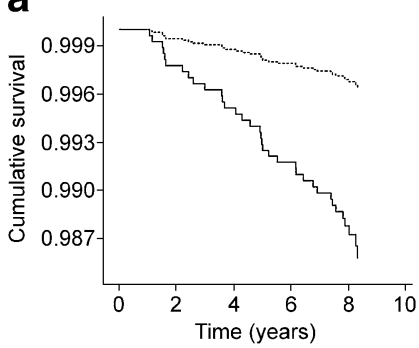

d

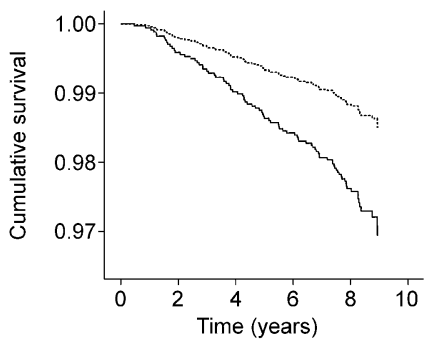

b

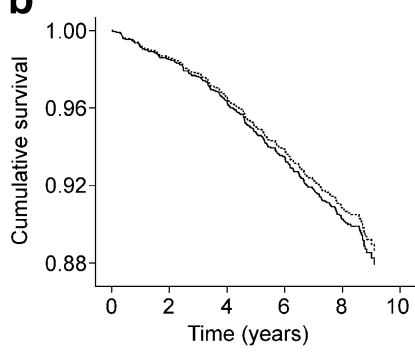

e

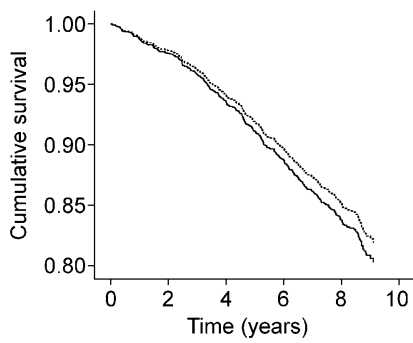

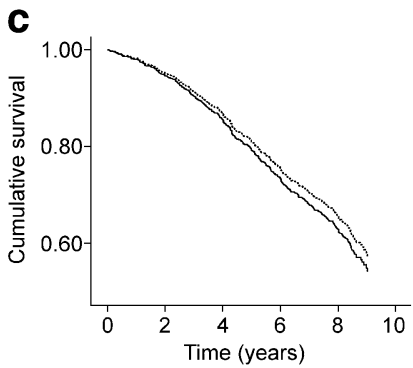

f

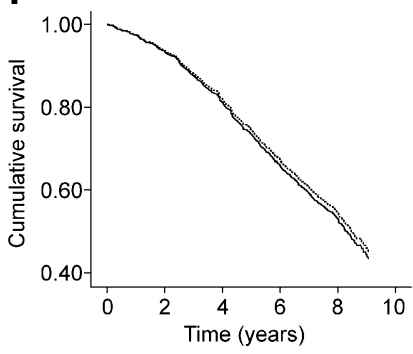


CVD mortality. In models adjusted for age (continuous within age groups) and sex, the HRs for non-CVD mortality were 1.47 (95\% CI: 0.84-2.57), 1.05 (0.79$1.40)$ and $1.03(0.80-1.34)$ in the three age groups, respectively, but with no significant interaction between metabolic syndrome and age $(p=0.56)$.

Second, using CVD alone as the underlying cause of death (excluding cases where CVD was merely a contributing cause of death), the number of CVD deaths was reduced from 585 to $470(29,172$ and 269 CVD deaths in the three age groups, respectively). In general, we observed similar results to those obtained using CVD as a contributing cause of death, with HRs 3.82 (1.82-8.09), 0.99 $(0.73-1.34)$ and $1.20(0.93-1.53)$ in the three age groups respectively, in models adjusted for age (continuous) and sex.

Third, using the 2005 NCEP criteria for metabolic syndrome, we found the syndrome was significantly associated with a twofold increased relative risk of CVD and all-cause mortality in participants aged 40-59 years, but not significantly associated with mortality after the age of 60 years. The lower HRs in the youngest age group when using the NCEP compared with the IDF criteria were consistent with no significant interactions between age and NCEP metabolic syndrome. However, when analyses were restricted to the sub-sample without self-reported CVD at baseline, we found a significant interaction between age and NCEP metabolic syndrome on the risk of CVD mortality $(p=0.039)$.

\section{Discussion}

In this large population-based study of Norwegian adults followed for up to 9.5 years, we observed that the relative risk of mortality associated with the metabolic syndrome was strongly related to age. A substantial higher relative risk of CVD and all-cause mortality was seen in individuals aged 40-59 years at baseline, but not in those who were older. In the youngest age group, the metabolic syndrome accounted for $20.7 \%$ of the risk of CVD death and for $14.2 \%$ of all deaths. Findings were similar in men and women and remained unchanged after further adjustment for other risk factors (physical activity, smoking, total cholesterol and depression). Results were largely independent of CVD being present at baseline and of the FRS.

The strengths of the present study include: (1) examination of a large, community-based sample; (2) a relative large number of events from a reliable national register of death causes; and (3) a range of data on somatic illnesses, mental symptoms, medications, lifestyle and health-related behaviour. The study also has limitations. First, blood samples were drawn $\geq 4-9$ hours after the last meal, rather than after overnight fasting as recommended. However, we adjusted the triacylglycerol and glucose levels before further analysis [7], a procedure unlikely to have generated any age-specific difference in associated risks of mortality. Second, we had no data on incident non-fatal CVD and type 2 diabetes, which are major aims for risk reduction when diagnosing the metabolic syndrome [5]. Third, because nearly all participants were white, generalisation of these results to other ethnic groups should be done with caution.

While several studies have suggested that the metabolic syndrome is a risk factor for CVD events and for all-cause mortality, much of the data on risk of mortality has come from populations consisting predominantly of younger or middle-aged individuals [2-4, 8-15]. Results from studies in elderly populations are more conflicting, with reports of no $[16,22,25]$ and a slightly increased $[17,23,26]$ risk of total mortality. Similarly, for CVD mortality, one study [16] found no increased risk, whereas others [17-21, 23, 25] have reported a slightly increased risk. Some of these studies also included risk of non-fatal CVD [16, 18, 19, 21, 23], some found increased cardiovascular risk in men only $[20,25]$ and one study found increased risk before, but not after the age of 75 years [21]. One study investigated mortality risk separately in middle-aged and elderly men in the same population and found that the syndrome predicted mortality at the age of 50 years but not consistently at the age of 70 years [24]. In general, however, there has been a lack of studies exploring whether the syndrome carries similar risks across age groups in the same population.

Our results are consistent with previous reports of increased risk of mortality from the metabolic syndrome in middle-aged individuals, but extend those findings by suggesting that there is no increased risk of mortality in elderly individuals in the same population. Furthermore, our study extends previous findings by calculating both HRs and PARs and also by including adjustment for FRS.

The FRS has been considered the reference standard for primary prevention of CVD events, but a recent review indicated limited accuracy [31]. To improve prediction, attempts have been made to add other risk indicators such as the metabolic syndrome, but so far this indicator has not been found to improve CVD risk prediction beyond the level achieved by the FRS [8, 14]. In this study, we found that the metabolic syndrome was associated with increased relative risk of mortality over and above the FRS. From previous reports, and because the metabolic syndrome does not include established CVD risk factors such as age and smoking, this finding was not expected a priori. This robust effect of the metabolic syndrome is most likely to be due to the independent strong effect of abdominal obesity, which is not included in the FRS because obesity was thought to affect risk through other risk factors [32]. 
The effect of abdominal obesity may also explain much of the observed age difference in the relative risk of mortality associated with the metabolic syndrome. This would be consistent with previous findings of a general decline in the obesity-mortality association with increasing age $[33,34]$. Other possible explanations for the age difference in mortality associated with the metabolic syndrome might be survival bias, lifestyle intervention and medical treatment for components of the metabolic syndrome. Although we lack data on the latter issues, we have no indications that CVD risk factors have received more attention among elderly than among younger patients in routine clinical practice.

The results of our study indicate that the current guidelines $[5,6]$ for management of the metabolic syndrome may be justified in middle-aged individuals. More work is needed before such guidelines are warranted in a large proportion of otherwise healthy elderly individuals.

Acknowledgements The Nord-Trøndelag Health Study is a collaboration between the HUNT Research Centre, Faculty of Medicine, Norwegian University of Science and Technology, the Norwegian Institute of Public Health and the Nord-Trøndelag County Council.

Duality of interest The authors declare that there is no duality of interest associated with the manuscript.

\section{References}

1. Eckel RH, Grundy SM, Zimmet PZ (2005) The metabolic syndrome. Lancet 365:1415-1428

2. Ford ES (2005) Risks for all-cause mortality, cardiovascular disease, and diabetes associated with the metabolic syndrome: a summary of the evidence. Diabetes Care 28:1769-1778

3. Galassi A, Reynolds K, He J (2006) Metabolic syndrome and risk of cardiovascular disease: a meta-analysis. Am J Med 119:812819

4. Obunai K, Jani S, Dangas GD (2007) Cardiovascular morbidity and mortality of the metabolic syndrome. Med Clin North Am 91:1169-1184

5. Alberti KG, Zimmet P, Shaw J (2006) Metabolic syndrome-a new world-wide definition. A consensus statement from the International Diabetes Federation. Diabet Med 23:469-480

6. Grundy SM, Cleeman JI, Daniels SR et al (2005) Diagnosis and management of the metabolic syndrome: an American Heart Association/National Heart, Lung, and Blood Institute Scientific Statement. Circulation 112:2735-2752

7. Hildrum B, Mykletun A, Hole T, Midthjell K, Dahl AA (2007) Age-specific prevalence of the metabolic syndrome defined by the International Diabetes Federation and the National Cholesterol Education Program: the Norwegian HUNT 2 study. BMC Public Health 7:220

8. Dekker JM, Girman C, Rhodes T et al (2005) Metabolic syndrome and 10-year cardiovascular disease risk in the Hoorn Study. Circulation 112:666-673

9. Eberly LE, Prineas R, Cohen JD et al (2006) Metabolic syndrome: risk factor distribution and 18-year mortality in the Multiple Risk Factor Intervention Trial. Diabetes Care 29:123-130
10. Juutilainen A, Lehto S, Ronnemaa T, Pyorala K, Laakso M (2006) Proteinuria and metabolic syndrome as predictors of cardiovascular death in non-diabetic and type 2 diabetic men and women. Diabetologia 49:56-65

11. Katzmarzyk PT, Janssen I, Ross R, Church TS, Blair SN (2006) The importance of waist circumference in the definition of metabolic syndrome: prospective analyses of mortality in men. Diabetes Care 29:404-409

12. Lorenzo C, Williams K, Hunt KJ, Haffner SM (2007) The National Cholesterol Education Program-Adult Treatment Panel III, International Diabetes Federation, and World Health Organization definitions of the metabolic syndrome as predictors of incident cardiovascular disease and diabetes. Diabetes Care 30:8-13

13. The DECODE Study Group; Qiao Q (2006) Comparison of different definitions of the metabolic syndrome in relation to cardiovascular mortality in European men and women. Diabetologia 49:2837-2846

14. Stern MP, Williams K, Gonzalez-Villalpando C, Hunt KJ, Haffner SM (2004) Does the metabolic syndrome improve identification of individuals at risk of type 2 diabetes and/or cardiovascular disease? Diabetes Care 27:2676-2681

15. Wilson PW, D'Agostino RB, Parise H, Sullivan L, Meigs JB (2005) Metabolic syndrome as a precursor of cardiovascular disease and type 2 diabetes mellitus. Circulation 112:30663072

16. Butler J, Rodondi N, Zhu Y et al (2006) Metabolic syndrome and the risk of cardiovascular disease in older adults. J Am Coll Cardiol 47:1595-1602

17. Hillier TA, Rizzo JH, Pedula KL et al (2005) Increased mortality associated with the metabolic syndrome in older women with diabetes. Diabetes Care 28:2258-2260

18. Holvoet P, Kritchevsky SB, Tracy RP et al (2004) The metabolic syndrome, circulating oxidized LDL, and risk of myocardial infarction in well-functioning elderly people in the Health, Aging, and Body Composition Cohort. Diabetes 53:1068-1073

19. Lawlor DA, Smith GD, Ebrahim S (2006) Does the new International Diabetes Federation definition of the metabolic syndrome predict CHD any more strongly than older definitions? Findings from the British Women's Heart and Health Study. Diabetologia 49:41-48

20. Maggi S, Noale M, Gallina P et al (2006) Metabolic syndrome, diabetes, and cardiovascular disease in an elderly Caucasian cohort: the Italian Longitudinal Study on Aging. J Gerontol A Biol Sci Med Sci 61:505-510

21. McNeill AM, Katz R, Girman CJ et al (2006) Metabolic syndrome and cardiovascular disease in older people: the Cardiovascular Health Study. J Am Geriatr Soc 54:1317-1324

22. Ravaglia G, Forti P, Maioli F et al (2006) Metabolic syndrome: prevalence and prediction of mortality in elderly individuals. Diabetes Care 29:2471-2476

23. Simons LA, Simons J, Friedlander Y, McCallum J (2007) Does a diagnosis of the metabolic syndrome provide additional prediction of cardiovascular disease and total mortality in the elderly? The Dubbo Study. Med J Aust 186:400-403

24. Sundström J, Riserus U, Byberg L, Zethelius B, Lithell H, Lind L (2006) Clinical value of the metabolic syndrome for long term prediction of total and cardiovascular mortality: prospective, population based cohort study. BMJ 332:878-882

25. Wang J, Ruotsalainen S, Moilanen L, Lepisto P, Laakso M, Kuusisto J (2007) The metabolic syndrome predicts cardiovascular mortality: a 13-year follow-up study in elderly non-diabetic Finns. Eur Heart J 28:857-864

26. Mozaffarian D, Kamineni A, Prineas RJ, Siscovick DS (2008) Metabolic syndrome and mortality in older adults: the Cardiovascular Health Study. Arch Intern Med 168:969-978 
27. Holmen J, Midthjell K, Krüger $\varnothing$ et al (2003) The NordTrøndelag Health Study 1995-97 (HUNT 2): objectives, contents, methods and participation. Nor J Epidemiol 13:19-32

28. Mykletun A, Overland S, Aarø LE, Liabø HM, Stewart R (2008) Smoking in relation to anxiety and depression: evidence from a large population survey: the HUNT study. Eur Psychiatry 23:77-84

29. Mykletun A, Bjerkeset O, Dewey M, Prince M, Overland S, Stewart R (2007) Anxiety, depression, and cause-specific mortality: the HUNT study. Psychosom Med 69:323-331

30. Third report of the National Cholesterol Education Program (NCEP) expert panel on detection, evaluation, and treatment of high blood cholesterol in adults (Adult Treatment Panel III) final report (2002). Circulation 106:3143-3421
31. Brindle P, Beswick A, Fahey T, Ebrahim S (2006) Accuracy and impact of risk assessment in the primary prevention of cardiovascular disease: a systematic review. Heart 92:17521759

32. Wilson PW, D'Agostino RB, Levy D, Belanger AM, Silbershatz H, Kannel WB (1998) Prediction of coronary heart disease using risk factor categories. Circulation 97:1837-1847

33. Adams KF, Schatzkin A, Harris TB et al (2006) Overweight, obesity, and mortality in a large prospective cohort of persons 50 to 71 years old. N Engl J Med 355:763-778

34. Flegal KM, Graubard BI, Williamson DF, Gail MH (2005) Excess deaths associated with underweight, overweight, and obesity. JAMA 293:1861-1867 